\title{
Empirical field mapping for gradient nonlinearity correction of multi-site diffusion weighted MRI
}

\author{
Colin B. Hansen*1, Baxter P. Rogers ${ }^{* 2,3}$, Kurt G. Schilling ${ }^{2}$, Vishwesh Nath ${ }^{1}$, \\ Justin A. Blaber ${ }^{4}$, Okan Irfanoglu ${ }^{5}$, Alan Barnett ${ }^{5}$, Carlo Pierpaoli ${ }^{5}$, \\ Adam W. Anderson ${ }^{2,3}$, Bennett A. Landman ${ }^{1,2,3,4}$ \\ ${ }^{1}$ Computer Science, Vanderbilt University, Nashville, TN, USA; \\ ${ }^{2}$ Department of Radiology and Radiological Sciences, Vanderbilt University Medical Center, \\ Nashville, TN USA; \\ ${ }^{3}$ Department of Biomedical Engineering, Vanderbilt University, Nashville, TN USA; \\ ${ }^{4}$ Electrical Engineering, Vanderbilt University, Nashville, TN, USA; \\ ${ }^{5}$ National Institute of Biomedical Imaging and Bioengineering, Bethesda MD USA; \\ Corresponding Author: \\ Colin Hansen \\ PhD Student \\ Computer Science, Vanderbilt University \\ Email: colin.b.hansen@vanderbilt.edu
}

\section{ACKNOWLEDGEMENTS}

This work was supported by the National Institutes of Health under award numbers R01EB017230 and T32EB001628, and in part by the National Center for Research Resources, Grant UL1 RR024975-01. The content is solely the responsibility of the authors and does not necessarily represent the official views of the NIH. 


\section{ABSTRACT}

2 Background: Achieving inter-site / inter-scanner reproducibility of diffusion weighted magnetic

3 resonance imaging (DW-MRI) metrics has been challenging given differences in acquisition

4 protocols, analysis models, and hardware factors.

5 Purpose: Magnetic field gradients impart scanner-dependent spatial variations in the applied

6 diffusion weighting that can be corrected if the gradient nonlinearities are known. However,

7 retrieving manufacturer nonlinearity specifications is not well supported and may introduce errors

8 in interpretation of units or coordinate systems. We propose an empirical approach to mapping the

9 gradient nonlinearities with sequences that are supported across the major scanner vendors.

10 Study Type: Prospective observational study

11 Subjects: A spherical isotropic diffusion phantom, and a single human control volunteer

12 Field Strength/Sequence: 3T (two scanners). Stejskal-Tanner spin echo sequence with b-values

13 of $1000,2000 \mathrm{~s} / \mathrm{mm}^{2}$ with 12,32 , and 384 diffusion gradient directions per shell.

14 Assessment: We compare the proposed correction with the prior approach using manufacturer

15 specifications against typical diffusion pre-processing pipelines (i.e., ignoring spatial gradient

16 nonlinearities). In phantom data, we evaluate metrics against the ground truth. In human and

17 phantom data, we evaluate reproducibility across scans, sessions, and hardware. 
18 Statistical Tests: Wilcoxon rank-sum test between uncorrected and corrected data.

19 Results: In phantom data, our correction method reduces variation in mean diffusivity across

20 sessions over uncorrected data $(\mathrm{p}<0.05)$. In human data, we show that this method can also reduce

21 variation in mean diffusivity across scanners $(\mathrm{p}<0.05)$.

22 Conclusion: Our method is relatively simple, fast, and can be applied retroactively. We advocate

23 incorporating voxel-specific b-value and b-vector maps should be incorporated in DW-MRI

24 harmonization preprocessing pipelines to improve quantitative accuracy of measured diffusion

25 parameters.

26 Keywords: Gradient Nonlinearity, Field Estimation, Pre-processing, DW-MRI 
29 Physics underlying magnetic resonance imaging (MRI) gradient coil designs result in nonuniform

30 magnetic field gradients during acquisition. This leads to spatial image warping [1-4] in magnetic

31 resonance images and gradient distortion in diffusion weighted magnetic resonance imaging (DW-

32 MRI) [5-9]. The introduced spatial variation can impact estimated diffusion tensor information

33 [10] or high-angular resolution diffusion measurements [11]. Bammer et al. show in extreme cases

34 the gradient nonuniformity can lead to an overestimation in the diffusion coefficient up to $30 \%$

35 and an underestimation up to $15 \%$ [12]. The severity of the effect increases with distance from the

36 magnet's isocenter [12] and with higher gradient amplitudes [12, 13]. The artifact becomes

37 especially troubling for multi-site studies that have varying scanner models and manufacturers [14]

38 and for studies utilizing very large gradient amplitudes such as in the human connectome project

39 (HCP) which utilized amplitudes up to $300 \mathrm{mT} / \mathrm{m}[13,15,16]$. Recent work has shown the effect

40 of gradient nonlinearities in the HCP cohort results in considerable bias in tractography results and

41 potentially incorrect interpretations in group-wise studies [17].

42 Various estimates of the coil magnetic field nonlinearities have been applied to improve accuracy

43 within and across sites [18-21]. An adaptive correction of diffusion information proposed by

44 Bammer et al. relies on calculating the spatially varying gradient coil $L$. This approach is achieved

45 by relating the actual gradients with the desired gradients [12], and has become standard practice

$46[22,23]$. However, this approach assumes that the gradient calibration specified by the

47 manufacturer is readily available. Spherical harmonics $(\mathrm{SH})$ based techniques are already

48 implemented by manufacturers in the scanning systems to account for the spatial image warping

49 effects of gradient nonlinearities [1, 24-27]. Yet, the spherical harmonic coefficients are not 
50 usually provided to regular users and may be subject to non-disclosure criteria. Additionally

51 gradient nonlinearity correction has been approached using noncartesian MR image reconstruction

$52[28]$.

53 To remove the need for the manufacturer supplied specifications, we demonstrate an empirical

54 field-mapping procedure which can be universally applied across platforms as defined by Rogers

55 et al. $[29,30]$. At two scanners (scanner A and scanner B), a large oil-filled phantom is used to

56 measure the magnetic field produced by each gradient coil. To estimate the achieved diffusion

57 gradient directions and b-values on a voxel-wise basis, solid harmonic basis functions are fit to the

58 measured magnetic field. The measured diffusivity (MD) and fractional anisotropy (FA) are

59 compared without nonlinearity correction, with nonlinearity correction using estimated fields, and

60 with nonlinearity correction using fields specified by the manufacturer for an ice-water diffusion

61 phantom. The reproducibility is compared between without nonlinearity correction and with

62 nonlinearity correction with the estimated fields for a subject scanned at two positions within the

63 scanner at scanner A. We show that our method removes the need for manufacturer specified

64 spherical harmonic coefficients and that the method reduces MD reproducibility error in-vivo

65 when the effect of gradient nonlinearities is present.

\section{METHODS}

\section{Measurement of gradient coil-generated magnetic fields}

68 Data were acquired across two 3T scanners: Scanner A and scanner B. Both of these are $94 \mathrm{~cm}$

69 bore Philips Intera Achieva MR whole-body systems and have a gradient strength of $80 \mathrm{mT} / \mathrm{m}$, a

$70200 \mathrm{~T} / \mathrm{m} / \mathrm{s}$ slew-rate. A phantom is used to estimate the gradient coil fields. The phantom is 24 
71 liters of a synthetic white oil (SpectraSyn 4 polyalphaolefin, ExxonMobil) in a polypropylene

72 carboy with an approximate diameter of $290 \mathrm{~mm}$ and height of 500mm [30]. This oil is used by the

73 manufacturer for some of their calibration phantoms which made it a reasonable choice. The

74 phantom was placed approximately at scanner isocenter and imaged with a dual echo GRE-based

75 field mapping sequence. Images are acquired at two echo times $1 \mathrm{~ms}$ apart, and the fieldmap is

76 computed from the phase difference of the two images. This follows the manufacturer's field

77 mapping and provides a field map with minimal phase wrapping or distortion. Four field maps

78 were acquired, one with shim field set to $0.05 \mathrm{mT} / \mathrm{m}$ on each axis $\mathrm{X}, \mathrm{Y}, \mathrm{Z}$ plus a final image with

79 gradient coil shim fields set to zero. Each used a $384 \mathrm{~mm}$ field of view with $4 \mathrm{~mm}$ isotropic voxel

80 size. Total scan time was approximately 5 minutes. Gradient coil fields were estimated by

81 subtracting the zero-shim field map from each coil's respective $0.05 \mathrm{mT} / \mathrm{m}$ field map. It should be

82 noted that the proposed method requires that the field maps are made using the same coils used to

83 produce the diffusion gradients, and systems that utilize gradient coil inserts may not be able to

84 directly utilize the technique. Field maps were acquired on 40 dates over the course of a year at

85 scanner B while scanner A only one session was acquired with the fieldmapping phantom.

86 For each coil, we modeled the magnetic field spatial variation as a sum of solid harmonics [12, 31,

87 32] to 7 th order, excluding even order terms due to the coils' physical symmetry. These basis

88 functions were fit to the field measurements with robust least squares, using all voxels within a

$89270 \mathrm{~mm}$ diameter sphere at isocenter. For comparison, the general shape of the human head is an

90 ellipsoid with an average height of 180 to $200 \mathrm{~mm}$ [33]. The result was an analytically differentiable

91 estimate of the true magnetic field produced by each gradient coil (Figure 1). This fitting procedure

92 was performed on an average field map derived from a series of scans to ensure stability. On 
93 Scanner B, the fitting procedure is also performed on the scanner manufacturer's estimate of the

94 coil fields as measured during manufacturing and installation. These are provided as a set of solid

95 harmonic functions and corresponding coefficients. The series of scans which are averaged are

96 defined for each subject session according to the closest 10 field map sessions in terms of date for

97 scanner B whereas 10 acquisitions were acquired within a single session at scanner A which are

98 averaged.

\section{Estimating achieved $\mathbf{b}$-values and gradient directions}

100

A spatially varying tensor $L$ relates the achieved magnetic field gradient to the intended one [12]:

101

$$
L=\left[\begin{array}{ccc}
\frac{\partial B_{Z}^{(x)}}{\partial x} & \frac{\partial B_{Z}^{(y)}}{\partial x} & \frac{\partial B_{Z}^{(z)}}{\partial x} \\
\frac{\partial B_{Z}^{(x)}}{\partial y} & \frac{\partial B_{Z}^{(y)}}{\partial y} & \frac{\partial B_{Z}^{(z)}}{\partial y} \\
\frac{\partial B_{Z}^{(x)}}{\partial z} & \frac{\partial B_{Z}^{(y)}}{\partial z} & \frac{\partial B_{Z}^{(z)}}{\partial z}
\end{array}\right]
$$

102 where $B_{z}^{(x)}$ is the $\mathrm{z}$ component of the magnetic field produced by unit amplitude of a nominal x-

103 gradient coil current, and similarly for $(\mathrm{y})$ and $(\mathrm{z})$. This tensor may be computed analytically from

104 the solid harmonic approximation to the measured field, then evaluated at spatial locations of

105 interest. We can use $L$ to relate the assumed gradient vector to the achieved gradient field and as

106 well as the assumed b-value to the achieved one. If we assume $|g|=1$ then the adjusted gradient

107 vector and b-value become:

$$
g^{\prime}=L g
$$


111 where $b^{\prime}$ is the adjusted b-value and $g^{\prime \prime}$ is the adjusted and normalized gradient vector. In the

112 common situation where the scanner reports the intended gradient direction and amplitude but the

113 full b-matrix [34-36] is not known, an approximate correction to adjust the signal $S_{i}$ for the $i^{\text {th }}$

114 diffusion acquisition relative to the reference signal $S_{0}$ is [18]:

$$
\ln \left(\frac{S_{i}}{S_{0}}\right)=-b g_{i}^{\prime T} D g_{i}^{\prime}=-b g_{i}^{T} L^{T} D L g_{i}
$$

116 where $b$ is the scalar b-value, $g$ is the intended gradient vector, $g^{\prime}$ is the actual gradient vector, and

$117 D$ is the diffusion tensor. If we substitute with $b^{\prime}$ and $g "$ equation 5 can be re-written as:

$$
\ln \left(\frac{s_{i}}{s_{0}}\right)=-b^{\prime} g_{i}^{\prime \prime} D g_{i}^{\prime \prime}=-b\left|g_{i}^{\prime}\right|^{2} \frac{g_{i}^{\prime T}}{\left|g_{i}^{\prime}\right|} D \frac{g_{i}^{\prime}}{\left|g_{i}^{\prime}\right|}=-b g_{i}^{T} L^{T} D L g_{i}
$$

119 Importantly, this is spatially varying and processing occurs voxelwise, but this may be used in any

120 desired way for further processing of the diffusion images. Figure 2 shows $L$ for each voxel

121 estimated using our empirical fieldmapping acquired on scanner B.

\section{EXPERIMENTS}

123 This section describes the set of analyses which aim to show the accuracy of the estimated fields

124 as well as their impact on resulting DW-MRI metrics in phantom and human data. All DW-MRI

125 are corrected for susceptibility distortion [37] and eddy current distortion [15] using FSL. 


\section{Empirically Estimated Fieldmaps}

127 Gradient nonlinearity correction is only viable if we can depend on the estimation to match the

128 true fields. To investigate if the magnitude estimated fieldmaps closely approximate the true fields,

129 we compare them to the fieldmaps specified by the manufacturer on scanner B. This was not done

130 for scanner A as the manufacturer specifications for scanner A were not provided. For comparison,

131 we take the average fieldmap from the latest 10 oil phantom scans on scanner B and calculate the

132 voxel-wise difference between this and the manufacturer specified fields. To evaluate the stability

133 of the empirical estimations, we report the variance across fields estimated from 40 individual oil

134 phantom scans acquired over time on scanner B. These additional acquisitions are unnecessary for

135 practical use and are strictly for evaluation purposes. Only a single acquisition would be needed

136 for this method to be deployed on a scanner to be applied to all previous and future acquisitions.

137 All evaluations on the empirical fields use a spherical mask with a radius of $135 \mathrm{~mm}$ from isocenter.

\section{Polyvinylpyrrolidone (PVP) phantom}

139 To evaluate the intra-scanner performance of the gradient field nonlinearity correction with the

140 empirical fieldmaps in a controlled environment, we use a 43\% Polyvinylpyrrolidone (PVP)

141 aqueous solution in a sealed spherical container that is $160 \mathrm{~mm}$ in diameter (PVP phantom) [38].

142 The PVP phantom is a large homogeneous material, and estimated metrics are expected to be the

143 same across the entire volume. Additionally, toxicology has shown PVP to be safe for use, and

144 PVP is stable and uniform. At scanner B, the phantom was scanned at three positions along the

145 magnet axis: superior ( $4 \mathrm{~cm}$ above isocenter), isocenter, and inferior ( $8 \mathrm{~cm}$ below isocenter). At

146 each position DWI data was acquired with diffusion weighting applied in twelve directions at a b-

147 value of $1000 \mathrm{~s} / \mathrm{mm}^{2}$ and twelve more were acquired at $2000 \mathrm{~s} / \mathrm{mm}^{2}$ with a TR of 7775, a voxel 
148 resolution of $2.5 \mathrm{~mm}$ by $2.5 \mathrm{~mm}$ by $2.5 \mathrm{~mm}$, and a FOV of $240 \mathrm{~mm}$ by $240 \mathrm{~mm}$ by $170 \mathrm{~mm}$.

149 Susceptibility distortion correction and eddy current distortion correction are applied without

150 movement correction. Signal to noise ratio (SNR) was calculated by fitting the signal to a tensor

151 in the phantom and taking the residuals after the fit. Using all diffusion volumes at each position,

152 MD is calculated without and with gradient nonlinearity correction using the empirically derived

153 fields and using the manufacturer specified fields. When calculating MD with the correction, the

154 estimated achieved b-values and gradient directions for each voxel are used. We report error in

155 terms of absolute percent error (APE) between each scan out of isocenter and the scan at isocenter.

156 All non-diffusion volumes to a structural T1 image using a rigid body transform restricted to only

157 use translations, and this registration is applied to the calculated MD before analysis.

\section{Human subject}

159 To evaluate the intra-scanner and inter-scanner performance of the gradient field nonlinearity 160 correction with the empirical fieldmaps in-vivo, we scanned a single subject at scanner A and

161 scanner B. At scanner B, two sessions were acquired of the subject with one session acquired with

162 the bridge of the subject's nose positioned at isocenter within the magnet and one session acquired

163 with the subject positioned $6 \mathrm{~cm}$ superior from isocenter. At scanner A, only one session is acquired

164 at isocenter. Each session consisted of twelve gradient directions at a b-value of $1000 \mathrm{~s} / \mathrm{mm}^{2}$,

165 twelve at a b-value of $2000 \mathrm{~s} / \mathrm{mm}^{2}$, a TR of $3700 \mathrm{~ms}$, a voxel resolution of $2.5 \mathrm{~mm}$ by $2.5 \mathrm{~mm}$ by

$1662.5 \mathrm{~mm}$, and a FOV of $240 \mathrm{~mm}$ by $240 \mathrm{~mm}$ by $170 \mathrm{~mm}$. Susceptibility distortion correction and eddy

167 current distortion correction are applied with movement correction for each session. Using all

168 diffusion volumes from each session, MD is calculated without and with gradient nonlinearity

169 correction using the empirically derived fields. At scanner B, MD is also calculated after correction 
170 with the manufacturer specifications. For analysis the scans are registered to a T1 acquired at

171 isocenter using FSL Flirt [39]. We report MD error as the absolute percent error between the two

172 scans acquired at scanner B and between the scan acquired at scanner A and the out of isocenter

173 scan acquired at scanner B.

174 We also evaluate the performance of the empirical correction with higher quality acquisitions on

175 scanner A. Again, two sessions are acquired of the subject: one with the bridge of the subject's 176 nose positioned at isocenter and one where the subject is shifted $4 \mathrm{~cm}$ inferior from isocenter. Each

177 session consisted of 384 gradient directions at a b-value of $1000 \mathrm{~s} / \mathrm{mm}^{2}$, a voxel resolution of

$1782.5 \mathrm{~mm}$ by $2.5 \mathrm{~mm}$ by $2.5 \mathrm{~mm}$, and a FOV of $240 \mathrm{~mm}$ by $240 \mathrm{~mm}$ by $170 \mathrm{~mm}$. Susceptibility distortion

179 correction and eddy current distortion correction are applied with movement correction for each

180 session. Using all diffusion volumes from each session, MD is calculated without and with gradient

181 nonlinearity correction using the empirically derived fields. For analysis the scans are registered

182 to a T1 acquired at isocenter using FSL Flirt [39]. We report MD error as the absolute percent error 183 between the two scans.

\section{RESULTS}

185 Empirically Estimated Fieldmaps

186 There are small differences between the manufacturer and the measured field produced by the

187 gradient coil. These are shown in Figure 1 in units of uT scaled by the intensity $(\mathrm{mT} / \mathrm{m})$ of the 188 applied gradient (uT/(mT/m), or mm). On average the difference at a given voxel is approximately

$1891 \mathrm{uT} /(\mathrm{mT} / \mathrm{m})$ in the $\mathrm{x}$ and $\mathrm{y}$ magnetic field gradients and $2 \mathrm{uT} /(\mathrm{mT} / \mathrm{m})$ in the $\mathrm{z}$ gradient field within

$190135 \mathrm{~mm}$ of isocenter. The difference maps indicate the presence of some structural artifacts. The 191 average standard deviation at a given voxel after 40 acquisitions acquired throughout a year is 
192 approximately $4 \mathrm{uT} /(\mathrm{mT} / \mathrm{m})$ in the $\mathrm{x}$ and $\mathrm{y}$ fields and $6 \mathrm{uT} /(\mathrm{mT} / \mathrm{m})$ in the $\mathrm{z}$ field within $135 \mathrm{~mm}$ of

193 isocenter.

194 PVP phantom

195 The mean absolute percent error within the phantom between the inferior scan and the isocenter

196 scan is approximately 5\% before correction. After correction using the manufacturer fields, this

197 falls to approximately $1.6 \%$. Correcting with the empirically derived fields leads to $0.9 \%$ mean

198 error. Figure 3 shows most of the error before correction in the inferior regions of the phantom

199 which were furthest from isocenter in the inferior scan.

200 When uncorrected, the mean absolute percent error within the phantom between the superior scan

201 and the isocenter scan is approximately 4.9\%. After correction using the manufacturer fields, this

202 falls to approximately $2 \%$. Correcting with the empirically derived fields leads to $1.3 \%$ mean error.

203 Figure 4 shows most of the error before correction in the superior regions of the phantom which

204 were furthest from isocenter in the superior scan.

\section{Human repositioned}

206 The intra-scanner sessions on scanner B result in a mean absolute percent error of 5.9\% before

207 correction within the brain volume excluding CSF regions. After correcting the scans using the

208 empirically estimated fields, the mean error is reduced to $5.6 \%$ and further to $5.4 \%$ if the

209 manufacturer specifications are used during correction. Just as in with the phantom, the error

210 attributable to the gradient nonlinearities before correction appears in the superior regions of the

211 brain which were furthest from isocenter during one of the sessions (Figure 5). 
212 For the inter-scanner experiment, the mean absolute percent error before correction is $7.2 \%$ and is

213 reduced $6.9 \%$ after correction using the estimated fields. Clearly the error that is accounted for in

214 the correction is the superior regions of the brain which were furthest from isocenter during the

215 session acquired on scanner B (Figure 6).

216 The intra-scanner sessions acquired on scanner A using a significantly higher number of gradient

217 directions results in a mean absolute percent error of $4.6 \%$ when no correction is applied. After

218 correction using the empirically estimated fields, the mean error is reduced to $4.2 \%$. The difference

219 can be seen in the inferior regions of the brain, specifically the cerebellum which was furthest from

220 isocenter during one of the sessions (Figure 7). Figure 8 shows the mean absolute percent error

221 across all voxels within the phantom and within the brain volume excluding cerebrospinal fluid

222 (CSF) regions for each method.

\section{DISCUSSION}

224 In comparing the empirically estimated fields to the fields specified by the manufacturer, we find

225 that our approximations are very similar. The largest differences are in the $\mathrm{z}$ gradient field which

226 corresponds to the largest variations in all the estimated fields across 40 oil phantom acquisitions.

227 In this study we use an average of fieldmaps across 10 acquisitions each acquired a week apart,

228 but this should not be necessary as the field produced by the gradient coil depends only on the coil

229 geometry and the current flowing in the coils. Unaltered system need only acquire the fields once

230 for this method, but further study on the stability of the empirical mapping may be necessary.

231 Additionally, further study on the stability of the fit of the spherical harmonics and the need for

232 higher order basis may be necessary. Appendix A shows the effects of different orders. 
233 The experiments with the PVP phantom show in a large isotropic volume the impact of the gradient

234 nonlinearities within the magnet and the effectiveness of the correction. The small superior shift

235 of $4 \mathrm{~cm}$ results in over $15 \%$ error in the superior voxels. In the case of a large inferior shift and a

236 smaller superior shift, the mean error is increased by a factor of two to five if these effects are not

237 accounted for. If we consider the experiments involving the human subject, we can see the impact

238 of this correction is reduced. This could in part due to imperfect registration which seems to have

239 contributed to error in the anterior regions of the brain. Results may vary depending on registration

240 strategy. We have tried multiple techniques with similar results. Though the absolute percent error

241 only changed by $0.3 \%$ to $0.4 \%$, some small regions see a similar magnitude of improvement, and

242 it is qualitatively clear that the correction is impacting regions we expect. The differences between

243 resulting absolute percent error using the empirical fields and the manufacturer fields is varies

244 between the phantom and the human subject. The results for the phantom indicate that the

245 estimated fields improve performance of the method, but the human subject results show a small

246 advantage for using the manufacturer field directly.

247 Though all intra-scanner results on scanner B are compared against using the manufacturer field

248 directly, future work should investigate the sensitivity of our proposed method and compare with

249 other field mapping methods such as proposed by Janke et. al [24] even though these methods

250 require that the manufacturer provide the solid harmonic coefficients. In recent work, another

251 approach is proposed for correcting voxel-wise b-value errors. Instead of correcting for gradient

252 nonlinearities in the coil, this method directly estimates a voxel-wise b-value map that is used to

253 correct resulting diffusion metrics [40]. While this method could account for errors that stem from

254 other sources of deviation than just gradient nonlinearities, the model requires an estimation of 
more parameters and likely it would be best practice to acquire a calibration scan along with every

256 subject acquisition. In comparison to apply the approach proposed in this work, only a single

257 calibration scan is necessary for each system.

258 While this method is successful in circumventing the need for manufacturer specifications which

259 are not always readily available, it should be noted that vendor-provided on-scanner gradient

260 nonlinearity correction is preferred for translation in a clinical environment. Additionally, when

261 working with any DICOM data coordinating world coordinate frame and patient frames can be

262 incredibly nuanced and should be considered carefully when applying any corrections post

263 acquisition. However, our approach remains as a solution to correct retroactively to enable the use

264 of acquired datasets which should be corrected for gradient nonlinearity effects for use in clinic

265 and in research.

\section{CONCLUSION}

267 This work shows that the errors caused by gradient nonlinearities is apparent in metrics derived

268 from DW-MRI but can be reduced using the correction outlined by Bammer et al. Using

269 empirically derived fields, we can achieve similar results without needing manufacturer

270 specification of the hardware. In both phantom and in-vivo data, error in MD can be significantly

271 reduced by applying this correction. We advocate for the use of gradient nonlinearity correction in

272 standard diffusion preprocessing pipelines and provide a simple method for empirically measuring

273 the fields necessary to account for the achieved b-values and b-vectors. 


\section{REFERENCES}

277

278

279

280

281

282

283

284

285

286

287

288

289

290

291

292

293

294

295

296

297

298

299

300

301

302

303

304

305

306

307

308

309

310

311

312

313

314

315

316

317

1. Glover, G.H. and N.J. Pelc, Method for correcting image distortion due to gradient nonuniformity. 1986, Google Patents.

2. Michiels, J., et al., On the problem of geometric distortion in magnetic resonance images for stereotactic neurosurgery. Magnetic resonance imaging, 1994. 12(5): p. 749-765.

3. Sumanaweera, T., et al., Quantifying MRI geometric distortion in tissue. Magnetic resonance in medicine, 1994. 31(1): p. 40-47.

4. Langlois, S., et al., MRI geometric distortion: a simple approach to correcting the effects of non-linear gradient fields. Journal of Magnetic Resonance Imaging: An Official Journal of the International Society for Magnetic Resonance in Medicine, 1999. 9(6): p. 821-831.

5. LeBihan, D. and R. Tumer, Diffusion and perfusion, magnetic resonance imaging, Mozbey Year Book. 1992, Inc.

6. Conturo, T.E., et al., Diffusion MRI: precision, accuracy and flow effects. NMR in Biomedicine, 1995. 8(7): p. 307-332.

7. Bernstein, M.A. and J.A. Polzin, Method and system for correcting errors in MR images due to regions of gradient non-uniformity for parametric imaging such as quantitative flow analysis. 2000, Google Patents.

8. Bammer, R., et al., Assessment of spatial gradient field distortion in diffusion-weighted imaging. Proceedings of the International Society for Magnetic Resonance in Medicine, Honolulu, HI, 2002: p. 1172.

9. Robson, M. Non-linear gradients on clinical MRI systems introduce systematic errors in $A D C$ and DTI measurements. in Proceedings of the 10th Annual Meeting of ISMRM, Honolulu. 2002.

10. Basser, P.J., Inferring microstructural features and the physiological state of tissues from diffusion-weighted images. NMR in Biomedicine, 1995. 8(7): p. 333-344.

11. Frank, L.R., Anisotropy in high angular resolution diffusion-weighted MRI. Magnetic Resonance in Medicine: An Official Journal of the International Society for Magnetic Resonance in Medicine, 2001. 45(6): p. 935-939.

12. Bammer, R., et al., Analysis and generalized correction of the effect of spatial gradient field distortions in diffusion-weighted imaging. Magnetic Resonance in Medicine: An Official Journal of the International Society for Magnetic Resonance in Medicine, 2003. 50(3): p. 560-569.

13. Setsompop, K., et al., Pushing the limits of in vivo diffusion MRI for the Human Connectome Project. Neuroimage, 2013. 80: p. 220-233.

14. Malyarenko, D.I., et al., Demonstration of nonlinearity bias in the measurement of the apparent diffusion coefficient in multicenter trials. J Magnetic resonance in medicine, 2016. 75(3): p. 1312-1323.

15. Andersson, J.L. and S.N. Sotiropoulos, An integrated approach to correction for offresonance effects and subject movement in diffusion MR imaging. Neuroimage, 2016. 125: p. 1063-1078.

16. McNab, J.A., et al., The Human Connectome Project and beyond: initial applications of $300 \mathrm{mT} / \mathrm{m}$ gradients. Neuroimage, 2013. 80: p. 234-245. 
17. Mesri, H.Y., et al., The adverse effect of gradient nonlinearities on diffusion MRI: From voxels to group studies. NeuroImage, 2019: p. 116127.

18. Tan, E.T., et al., Improved correction for gradient nonlinearity effects in diffusion-weighted imaging. Journal of Magnetic Resonance Imaging, 2013. 38(2): p. 448-453.

19. Newitt, D.C., et al., Gradient nonlinearity correction to improve apparent diffusion coefficient accuracy and standardization in the american college of radiology imaging network 6698 breast cancer trial. Journal of Magnetic Resonance Imaging, 2015. 42(4): p. 908-919.

20. Malyarenko, D.I., B.D. Ross, and T.L. Chenevert, Analysis and correction of gradient nonlinearity bias in apparent diffusion coefficient measurements. Magnetic resonance in medicine, 2014. 71(3): p. 1312-1323.

21. Malyarenko, D.I. and T.L. Chenevert, Practical estimate of gradient nonlinearity for implementation of apparent diffusion coefficient bias correction. Journal of Magnetic Resonance Imaging, 2014. 40(6): p. 1487-1495.

22. Sotiropoulos, S.N., et al., Advances in diffusion MRI acquisition and processing in the Human Connectome Project. Neuroimage, 2013. 80: p. 125-143.

23. Glasser, M.F., et al., The minimal preprocessing pipelines for the Human Connectome Project. Neuroimage, 2013. 80: p. 105-124.

24. Janke, A., et al., Use of spherical harmonic deconvolution methods to compensate for nonlinear gradient effects on MRI images. Magnetic Resonance in Medicine: An Official Journal of the International Society for Magnetic Resonance in Medicine, 2004. 52(1): p. 115-122.

25. Doran, S.J., et al., A complete distortion correction for MR images: I. Gradient warp correction. Physics in Medicine \& Biology, 2005. 50(7): p. 1343.

26. Tao, S., et al., Integrated image reconstruction and gradient nonlinearity correction. Magnetic resonance in medicine, 2015. 74(4): p. 1019-1031.

27. Tao, A.T., et al., Improving apparent diffusion coefficient accuracy on a compact $3 T$ MRI scanner using gradient nonlinearity correction. Journal of Magnetic Resonance Imaging, 2018. 48(6): p. 1498-1507.

28. Tao, S., et al., NonCartesian MR image reconstruction with integrated gradient nonlinearity correction. Medical physics, 2015. 42(12): p. 7190-7201.

29. Rogers, B.P., et al. Phantom-based field maps for gradient nonlinearity correction in diffusion imaging. in Medical Imaging 2018: Physics of Medical Imaging. 2018. International Society for Optics and Photonics.

30. Rogers, B.P., et al. Stability of gradient field corrections for quantitative diffusion MRI. in Medical Imaging 2017: Physics of Medical Imaging. 2017. International Society for Optics and Photonics.

31. Tough, R.J. and A.J. Stone, Properties of the regular and irregular solid harmonics. Journal Of Physics A: Mathematical General, 1977. 10(8): p. 1261.

32. Caola, M., Solid harmonics and their addition theorems. Journal of Physics A: Mathematical General, 1978. 11(2): p. L23.

33. Makris, N., et al., MRI-based anatomical model of the human head for specific absorption rate mapping. Medical \& biological engineering \& computing, 2008. 46(12): p. 12391251. 
34. Mattiello, J., P.J. Basser, and D. Le Bihan, The b matrix in diffusion tensor echo-planar imaging. Magnetic Resonance in Medicine, 1997. 37(2): p. 292-300.

35. Mattiello, J., P.J. Basser, and D. LeBihan, Analytical expressions for the b matrix in NMR diffusion imaging and spectroscopy. Journal of magnetic resonance, Series A, 1994. 108(2): p. 131-141.

36. Alger, J.R., The diffusion tensor imaging toolbox. Journal of Neuroscience, 2012. 32(22): p. 7418-7428.

37. Andersson, J.L., S. Skare, and J. Ashburner, How to correct susceptibility distortions in spin-echo echo-planar images: application to diffusion tensor imaging. Neuroimage, 2003. 20(2): p. 870-888.

38. Pierpaoli, C., et al. Polyvinylpyrrolidone (PVP) water solutions as isotropic phantoms for diffusion MRI studies. in Proc Intl Soc Magn Reson Med. 2009.

39. Jenkinson, M., et al., Improved optimization for the robust and accurate linear registration and motion correction of brain images. Neuroimage, 2002. 17(2): p. 825-841.

40. Lee, Y., et al., A comprehensive approach for correcting voxel-wise b-value errors in diffusion MRI. 2019. 
bioRxiv preprint doi: https://doi.org/10.1101/2020.05.18.102558; this version posted November 16, 2020. The copyright holder for this preprint (which was not certified by peer review) is the author/funder, who has granted bioRxiv a license to display the preprint in perpetuity. It is made available under aCC-BY-NC-ND 4.0 International license.

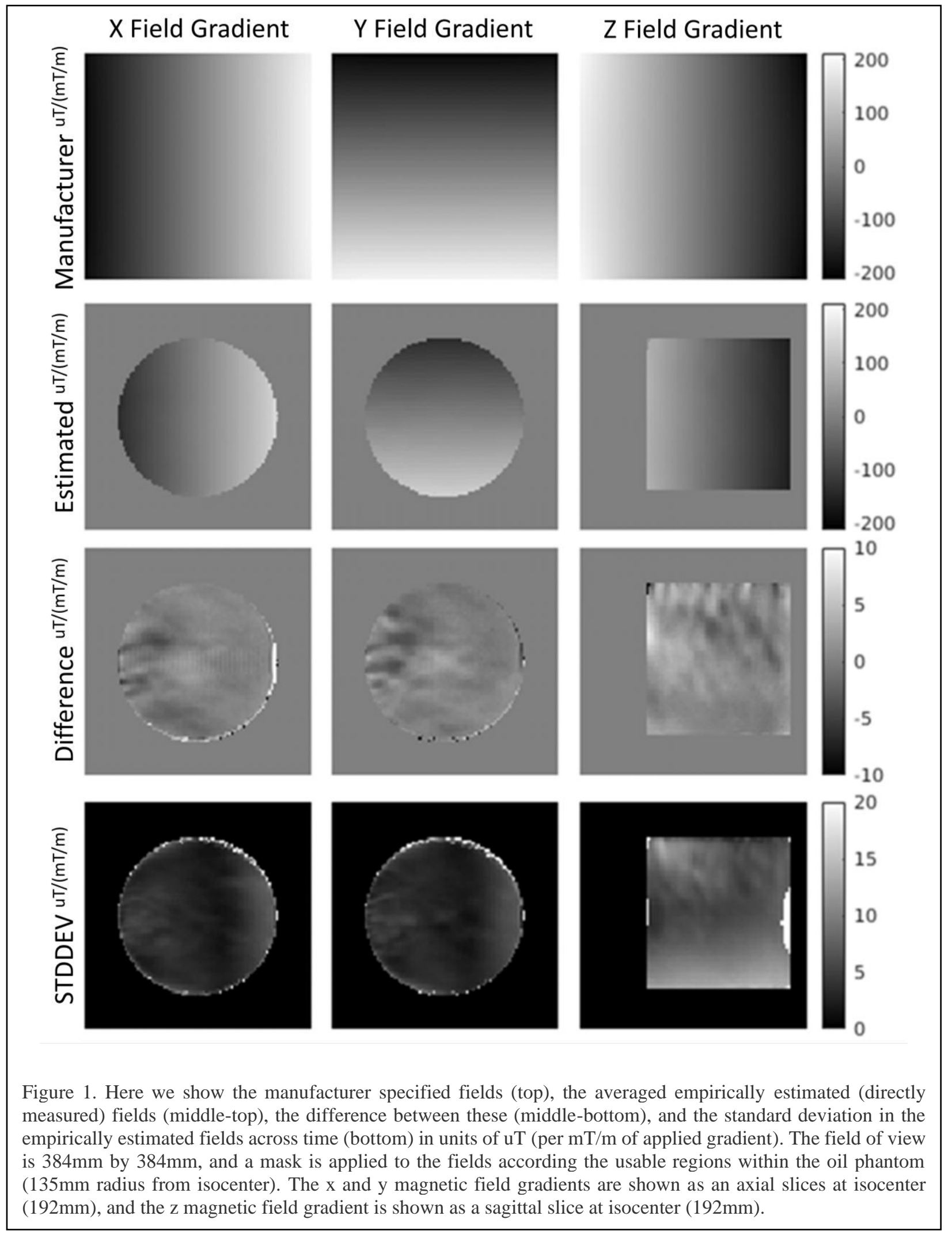


bioRxiv preprint doi: https://doi.org/10.1101/2020.05.18.102558; this version posted November 16, 2020. The copyright holder for this preprint (which was not certified by peer review) is the author/funder, who has granted bioRxiv a license to display the preprint in perpetuity. It is made available under aCC-BY-NC-ND 4.0 International license.

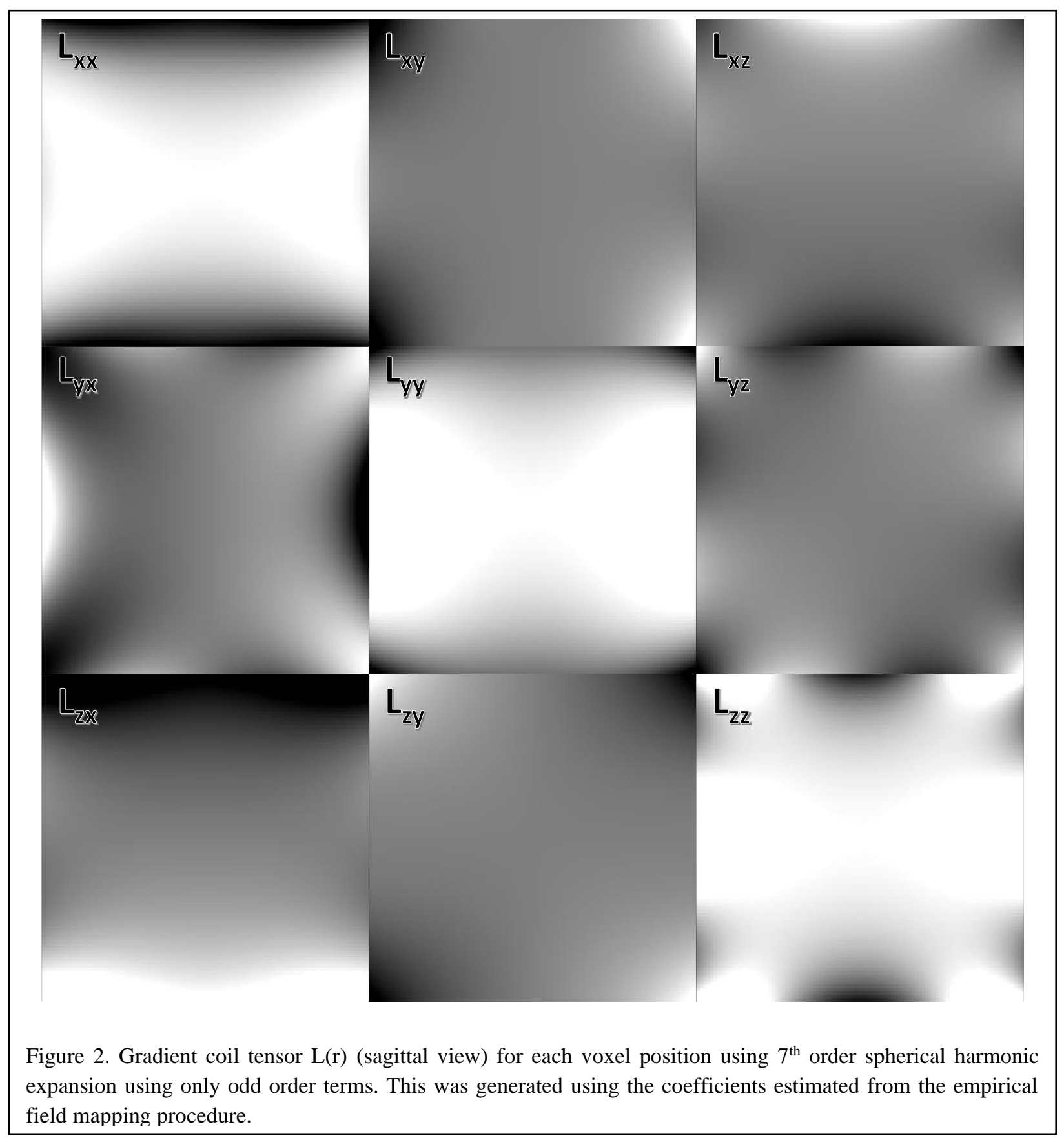


bioRxiv preprint doi: https://doi.org/10.1101/2020.05.18.102558; this version posted November 16, 2020. The copyright holder for this Preprint (which was not certified by peer review) is the author/funder, who has granted bioRxiv a license to display the preprint in perpetuity. It is made available under aCC-BY-NC-ND 4.0 International license.

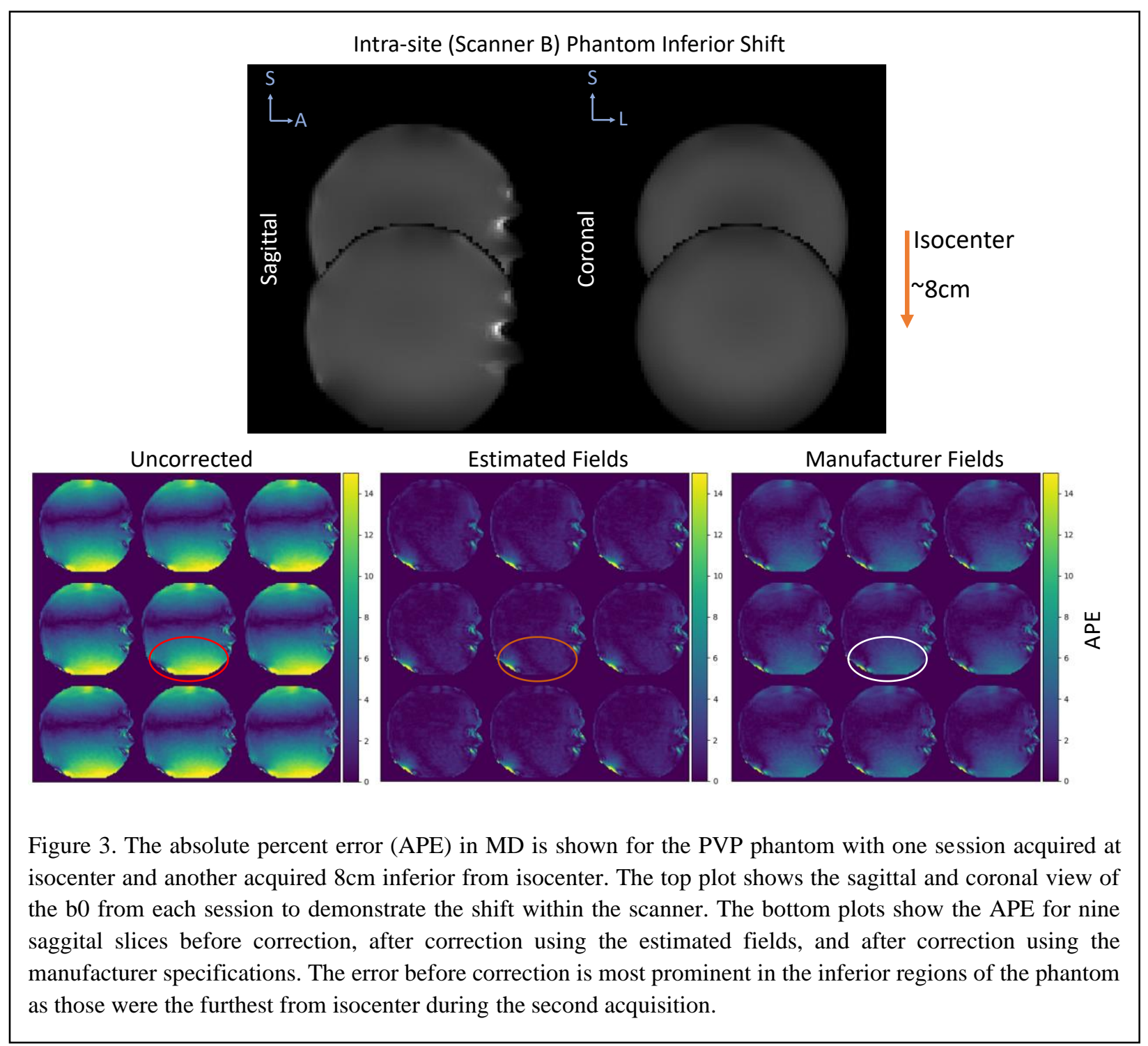


bioRxiv preprint doi: https://doi.org/10.1101/2020.05.18.102558; this version posted November 16, 2020. The copyright holder for this Preprint (which was not certified by peer review) is the author/funder, who has granted bioRxiv a license to display the preprint in perpetuity. It is made available under aCC-BY-NC-ND 4.0 International license.

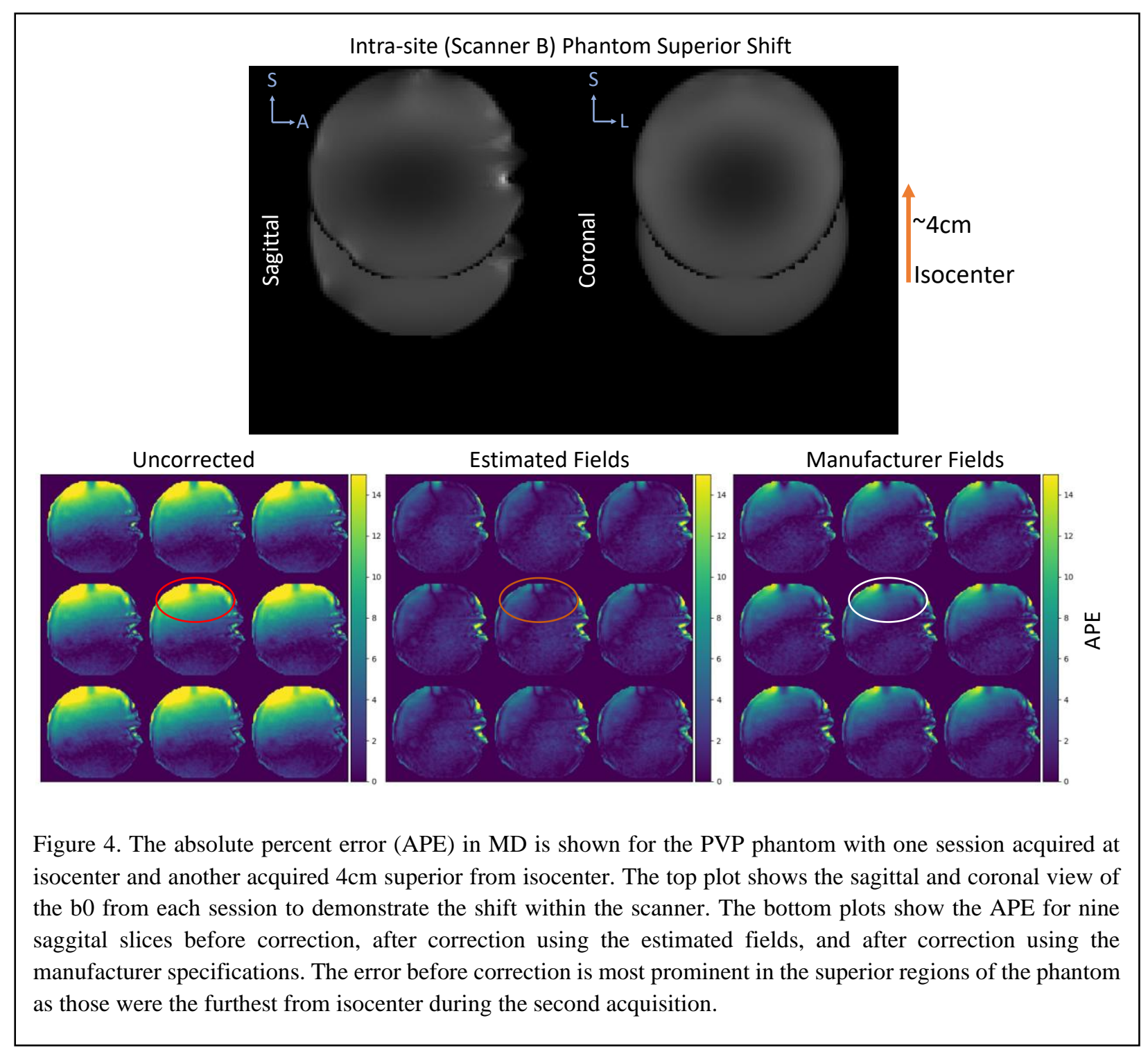


bioRxiv preprint doi: https://doi.org/10.1101/2020.05.18.102558; this version posted November 16, 2020. The copyright holder for this Breprint (which was not certified by peer review) is the author/funder, who has granted bioRxiv a license to display the preprint in perpetuity. It is made available under aCC-BY-NC-ND 4.0 International license.

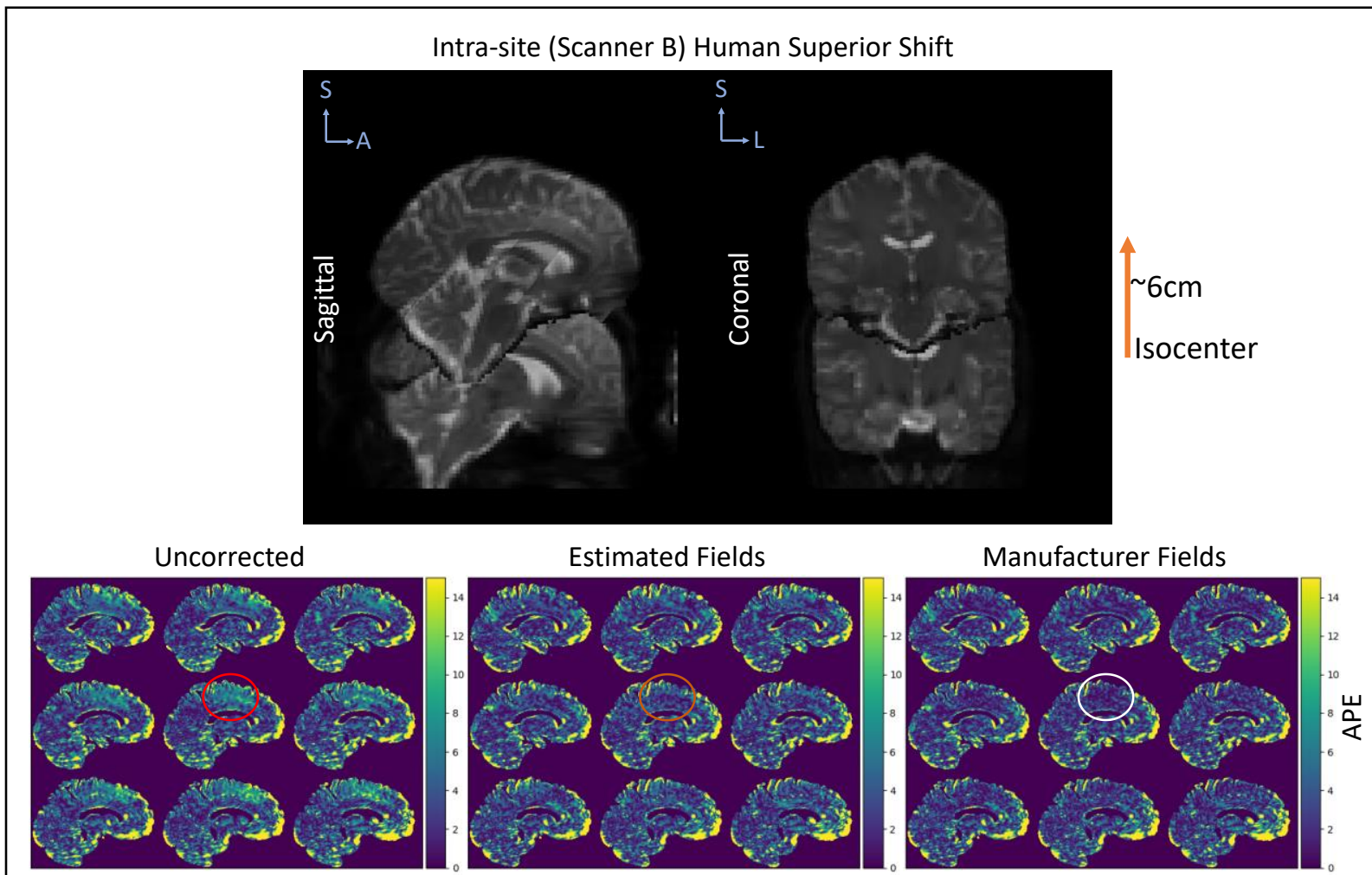

Figure 5. The absolute percent error (APE) in MD is shown for the human subject with one session acquired at isocenter and another acquired $6 \mathrm{~cm}$ superior from isocenter on scanner B. The top plot shows the sagittal and coronal view of the $\mathrm{b} 0$ from each session to demonstrate the shift within the scanner. The bottom plots show the APE for nine saggital slices before correction, after correction using the estimated fields, and after correction using the manufacturer specifications. The error before correction is most prominent in the superior regions of the phantom as those were the furthest from isocenter during the second acquisition. 
bioRxiv preprint doi: https://doi.org/10.1101/2020.05.18.102558; this version posted November 16, 2020. The copyright holder for this prëprint (which was not certified by peer review) is the author/funder, who has granted bioRxiv a license to display the preprint in perpetuity. It is made available under aCC-BY-NC-ND 4.0 International license.

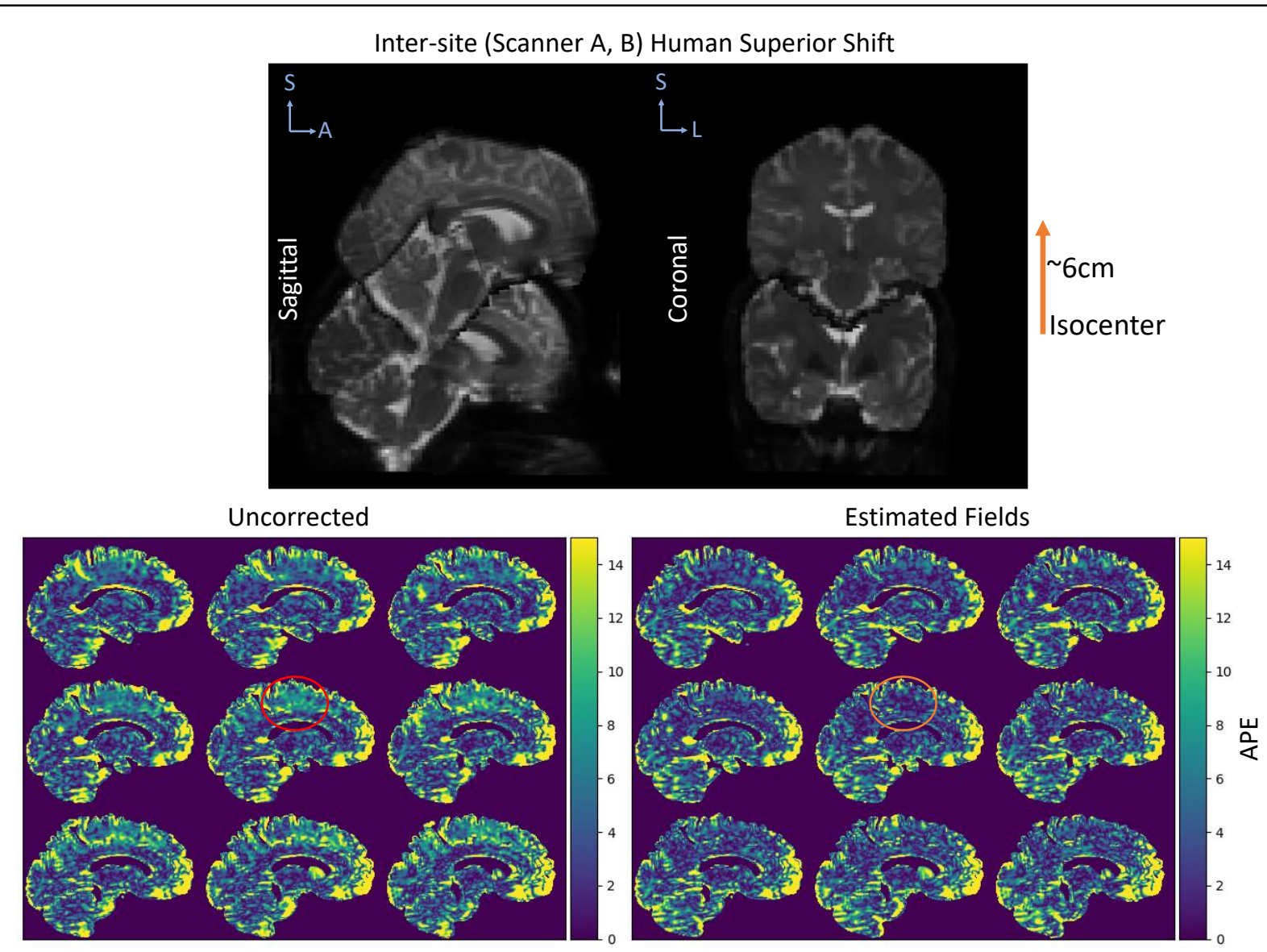

Figure 6. The absolute percent error (APE) in MD is shown for the human subject with one session acquired at isocenter on scanner $\mathrm{A}$ and another acquired $6 \mathrm{~cm}$ superior from isocenter on scanner B. The top plot shows the sagittal and coronal view of the b0 from each session to demonstrate the shift within the scanner. The bottom plots show the APE for nine saggital slices before correction, after correction using the estimated fields, and after correction using the manufacturer specifications. The error before correction is most prominent in the superior regions of the phantom as those were the furthest from isocenter during the second acquisition. 
bioRxiv preprint doi: https://doi.org/10.1101/2020.05.18.102558; this version posted November 16, 2020. The copyright holder for this preprint (which was not certified by peer review) is the author/funder, who has granted bioRxiv a license to display the preprint in perpetuity. It is made available under aCC-BY-NC-ND 4.0 International license.

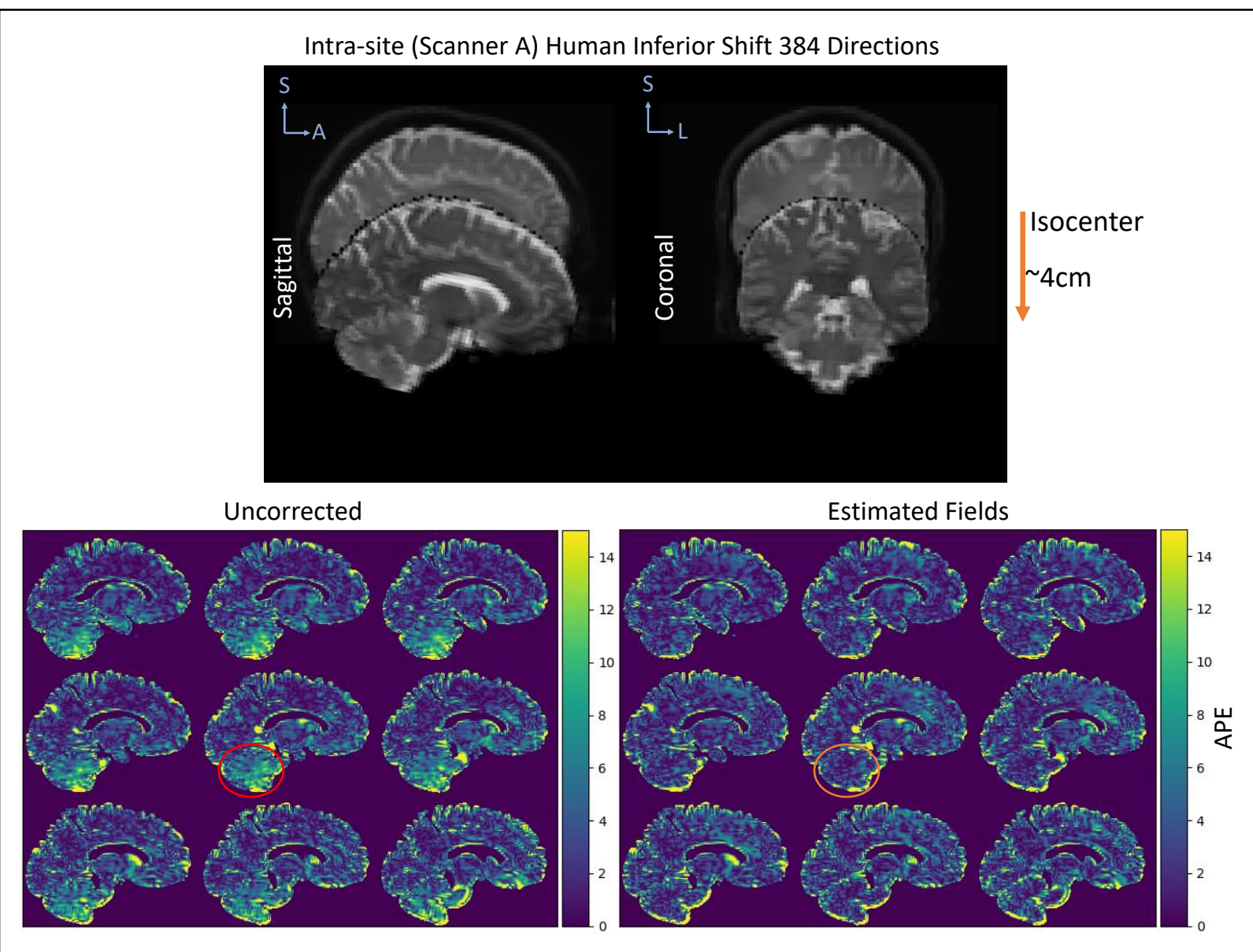

Figure 7. The absolute percent error (APE) in MD is shown for the human subject with one session acquired at isocenter and another acquired $4 \mathrm{~cm}$ inferior from isocenter on scanner A. These acquisitions were acquired with 384 directions. The top plot shows the sagittal and coronal view of the b0 from each session to demonstrate the shift within the scanner. The bottom plots show the APE for nine saggital slices before correction, after correction using the estimated fields, and after correction using the manufacturer specifications. The error before correction is most prominent in the inferior regions of the phantom as those were the furthest from isocenter during the second acquisition. 
bioRxiv preprint doi: https://doi.org/10.1101/2020.05.18.102558; this version posted November 16, 2020. The copyright holder for this Preprint (which was not certified by peer review) is the author/funder, who has granted bioRxiv a license to display the preprint in perpetuity. It is made available under aCC-BY-NC-ND 4.0 International license.

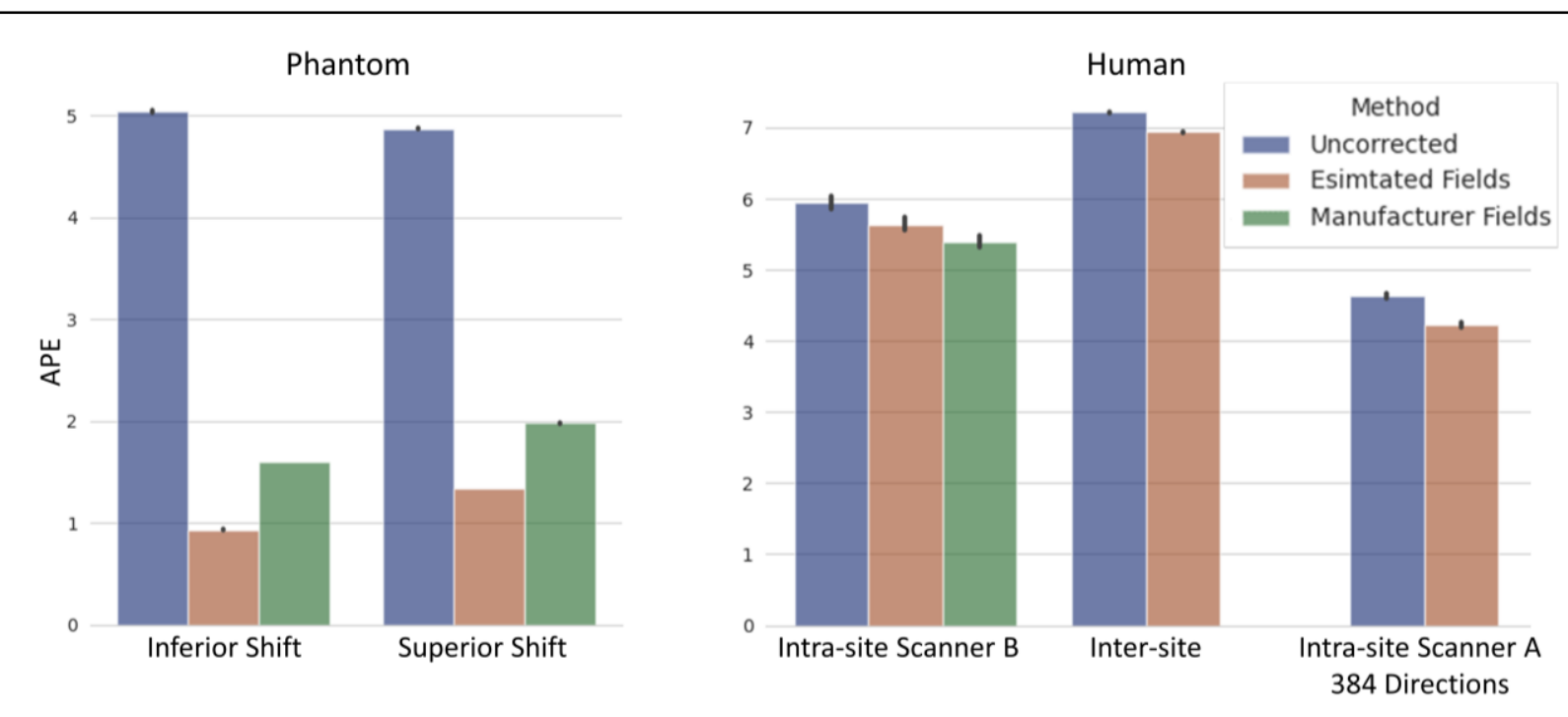

Figure 8. The mean APE within the phantom and brain excluding CSF regions are shown for each experiment without correction, after correction with the estimated fieldmaps, and after correction with the manufacturer specifications when available. 
bioRxiv preprint doi: https://doi.org/10.1101/2020.05.18.102558; this version posted November 16, 2020. The copyright holder for this PrEprint (which was not certified by peer review) is the author/funder, who has granted bioRxiv a license to display the preprint in perpetuity. It is made available under aCC-BY-NC-ND 4.0 International license.

\section{APPENDIX A}

392 The PVP phantom is corrected using fieldmaps estimated with various orders of solid harmonics.

393 Regardless of the order, both FA and MD reproducibility errors decrease when compared to the

394 uncorrected error. However, we find that a $3^{\text {rd }}$ order basis results in the lowest FA error but a higher

395 MD error. Between the higher order basis, the $7^{\text {th }}$ order solid harmonics achieves lower FA error.
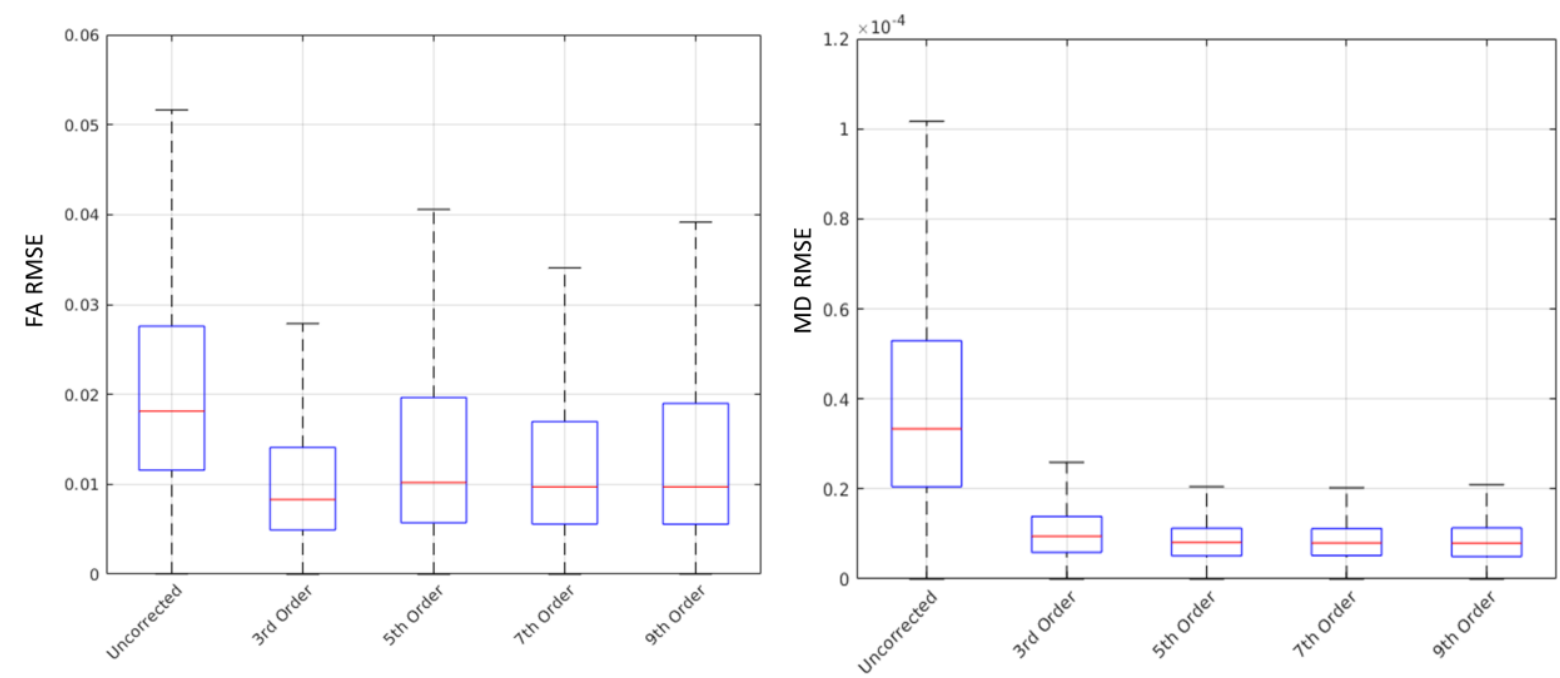

Figure A.1: The reproducibility error in FA and MD for the PVP phantom are calculated using the estimated fieldmap utilizing different orders of solid harmonics. Orders higher than $3^{\text {rd }}$ achieve lower MD RMSE but tend to have higher FA RMSE. 